\title{
Das BMW-Modell: Neukeynesianische monetäre Makroökonomie für die Lehre
}

\author{
Peter Bofinger, \\ Eric Mayer, \\ Timo Wollmershäuser
}

Trotz zunehmender Kritik wird in den meisten Lehrbüchern das IS/LM-AS/AD-Modell noch immer als das Standardmodell der Makroökonomie darstellt. In einer Reihe von Beiträgen haben Bofinger, Mayer und Wollmershäuser (2002a, 2002b, 2003a und 2003b) einen alternativen Modelrahmen, das sog. BMW-Modell, präsentiert, der den Ansatz von Romer (2000) in ein einfaches, aber überzeugendes makroökonomisches Model überführt. Trotz seiner Einfachheit eignet sich das BMW-Modell zur Diskussion relevanter Fragestellungen wie etwa dem Konzept des Inflation Targeting, geldpolitischer Regelbindung oder der Glaubwürdigkeit von Geldpolitik. Dieser Beitrag fasst die wichtigsten Elemente des BMW-Modells zusammen.

\section{Einleitung}

Die makroökonomische Lehre wird heute nach wie vor durch das IS/LM-AS/AD-Modell dominiert - und dies trotz vielfacher Kritik. Während Colander (1995) darauf verwies, dass die Erklärung des aggregierten Angebots im Rahmen des IS/LM-AS/AD-Modells inkonsistent ist, zeigte Romer (2000), dass es nur schlecht geeignet ist, Geldpolitik in Form einer Zinssteuerung zu beschreiben. Walsh (2002) hat darüber hinaus kritisiert, dass eine Politik des „Inflation Targeting“ durch das Modell nicht abgebildet werden kann. Der vorliegende Beitrag bietet ein leicht verständliches und gleichzeitig umfassendes Modell, das auf dem Ansatz von Romer 
(2000) aufbaut. Obwohl es einfach gehalten ist, enthält es wichtige Aspekte der Neukeynesianischer Makroökonomie (vgl. Clarida, Gali und Gertler, 1999) und ermöglicht gleichzeitig die Darstellung neuerer Ansätze der Geldpolitik, wie z. B. Inflation Targeting, Regelbindung der Notenbank, Erwartungen der privaten Haushalte und Glaubwürdigkeit der Notenbank. Es bildet die Geldpolitik im Rahmen einer Zinssteuerung ab und konzentriert sich auf die Reaktion der Notenbank bei Angebots- und Nachfrageschocks.

\section{Das BMW-Modell}

\subsection{Die Bausteine und Annahmen des Modells}

Das Modell besteht aus drei Bausteinen:

- eine vom Realzins abhängige gesamtwirtschaftliche Nachfragekurve (IS Kurve)

- eine Phillipskurve und

- $\quad$ eine geldpolitische Regel.

Die IS Kurve beschreibt die Outputlücke $\mathrm{y}$ in Abhängigkeit von autonomen Nachfragekomponenten a, dem kurzfristigen Realzins $r$ und einem Nachfrageschock $\varepsilon_{1}$ :

$$
\mathrm{y}=\mathrm{a}-\mathrm{br}+\varepsilon_{1},
$$

wobei die Outputlücke als die prozentuale Abweichung des gesamtwirtschaftlichen Einkommens vom Potentialoutput definiert ist. Aus Gleichung (1) wird ersichtlich, dass die Outputlücke alleine vom Realzins abhängt, solange keine Nachfrageschocks auftreten. Nachfrageschocks sind definiert als temporäre Störung der autonomen Komponenten, der Staatsausgaben, der Investitionen sowie des Konsums. Bei gegebenen Modellparametern a und b ist der Realzins, bei welchem die Outputlücke null ist, der so genannte neutrale kurzfristige Realzins $\mathrm{r}_{0}$ (vgl. Blinder, 1998, S. 31):

$$
\mathrm{r}_{0}=\frac{\mathrm{a}}{\mathrm{b}}
$$

Der zweite Baustein des Modells ist die um Erwartungen erweiterte Phillipskurve: 


$$
\pi=\pi^{\mathrm{e}}+\mathrm{dy}+\varepsilon_{2}
$$

Demnach wird die Inflationsrate durch die Inflationserwartungen $\pi^{\mathrm{e}}$, die Outputlücke y und einen Angebotsschock $\varepsilon_{2}$ determiniert. Angebotsschocks sind zum Beispiel definiert als Abweichung des Reallohnanstiegs vom Produktivitätszuwachs oder als eine unerwartete Veränderung der Ölpreise. Der Einfachheit halber wird zunächst angenommen, dass die Notenbank glaubwürdig ist. Deshalb entsprechen die Inflationserwartungen $\pi^{\mathrm{e}}$ dem Inflationsziel $\pi_{0}$, und die Phillipskurve kann vereinfacht folgendermaßen dargestellt werden:

$$
\pi=\pi_{0}+\mathrm{dy}+\varepsilon_{2} .
$$

Gleichung (4) kann als ein Spezialfall interpretiert werden, wonach Inflationserwartungen rational gebildet werden und die aktuelle Inflationsrate durch die zukünftig erwartete Inflationsrate determiniert wird. Die Herleitung dieser Vereinfachung präsentieren wir in Kapitel 2.6 .

Bei der Formulierung der geldpolitischen Regel wird im Rahmen des BMW-Modells angenommen, dass die Notenbank den Realzins steuert. Zwar hat die Notenbank in der Realität nur vollständige Kontrolle über den Nominalzins auf dem Geldmarkt, aber indirekt steuert sie den Realzins, solange sie in der Lage ist, die Wirkung ihrer Politik auf die Inflationsrate im voraus $\mathrm{zu}$ antizipieren. In einer Welt mit rationalen Erwartungen ist diese Vorraussetzung gegeben. In der einfachsten Variante des BMW-Modells wird angenommen, dass die Notenbank den Zinssatz ohne eine Form der Regelbindung festlegt, also diskretionäre Geldpolitik betreibt. Das vereinfacht die grafische Darstellung der Wirkung geldpolitischer Maßnahmen (vgl. Kapitel 2.2). In der Realität verfolgen Notenbanken meistens eine geldpolitische Strategie. So entschieden sich im Laufe der 90er eine Reihe führender Zentralbanken (z.B. die Bank of England, die Reserve Bank of New Zealand und die Bank of Canada) für die Strategie des Inflation Targeting (vgl. Kapitel 2.3). Dazu verwendet die Notenbank alle relevanten Informationen und bestimmt den optimalen Zinssatz anhand einer Verlustfunktion, in der ihre Präferenzen hinsichtlich des Inflationsziels sowie eines stabilen Outputs abgebildet werden (vgl. Svensson, 1999). Alternativ dazu könnte die Notenbank ihren Zinssatz mittels einer Heuristik festlegen (vgl. Kapitel 2.4). Im Gegensatz zum Inflation Targeting ist für eine Heuristik 
charakteristisch, dass die Notenbank nur aufgrund von wenigen ausgewählten Informationen eine Entscheidung trifft. Die bekannteste Heuristik ist die Taylor Regel (Taylor, 1993).

Ähnlich wie im IS/LM-AS/AD-Modell sind auch im BMW-Ansatz zur grafischen Analyse von geldpolitischen Massnahmen zwei Diagramme notwendig (Abb. 1). Die gesamtwirtschaftliche Nachfragekurve und die Zinslinie der Notenbank werden im r-y-Raum abgebildet. Die gesamtwirtschaftliche Nachfragekurve hat eine negative Steigung (vgl. Gleichung (1)), und die Zinslinie der Notenbank wird im Falle einer diskretionären Geldpolitik durch eine Horizontale dargestellt. Im gesamtwirtschaftlichen Gleichgewicht befindet sich der Schnittpunkt der gesamtwirtschaftlichen Nachfragekurve mit der Zinslinie an der Stelle, an der die Outputlücke null ist. Die Phillipskurve weist im $\pi$-y-Raum eine positive Steigung auf (vgl. Gleichung (4)). Wenn die Outputlücke null ist, entspricht die Inflationsrate der Zielinflationsrate.

\subsection{Diskretionäre Geldpolitik}

Tritt ein negativer Nachfrageschock $\left(\varepsilon_{1}<0\right)$ auf, verschiebt sich die IS-Kurve nach unten (vgl. Abb. 1). Bei einem konstantem Realzins tritt jetzt eine negative Outputlücke $\left(\mathrm{y}_{1}<0\right)$ auf, die im unteren Teil der Abb. die Inflationsrate auf $\pi_{1}$, und somit unter die Zielinflationsrate $\pi_{0}$ der Notenbank, sinken lässt. Wenn nun die Notenbank den Realzins von $r_{0}$ auf $r_{1}$ senkt, ist die Outputlücke wieder geschlossen und die Inflationsrate entspricht der Zielinflationsrate. Die entscheidende Erkenntnis ist, dass im Fall eines Nachfrageschocks kein Zielkonflikt zwischen der Stabilisierung des Outputs und der Inflationsrate besteht, da die Zinserhöhung sowohl die ökonomischer Aktivität wieder belebt, als auch die Inflationsrate zurück zu ihrem Zielwert führt. 


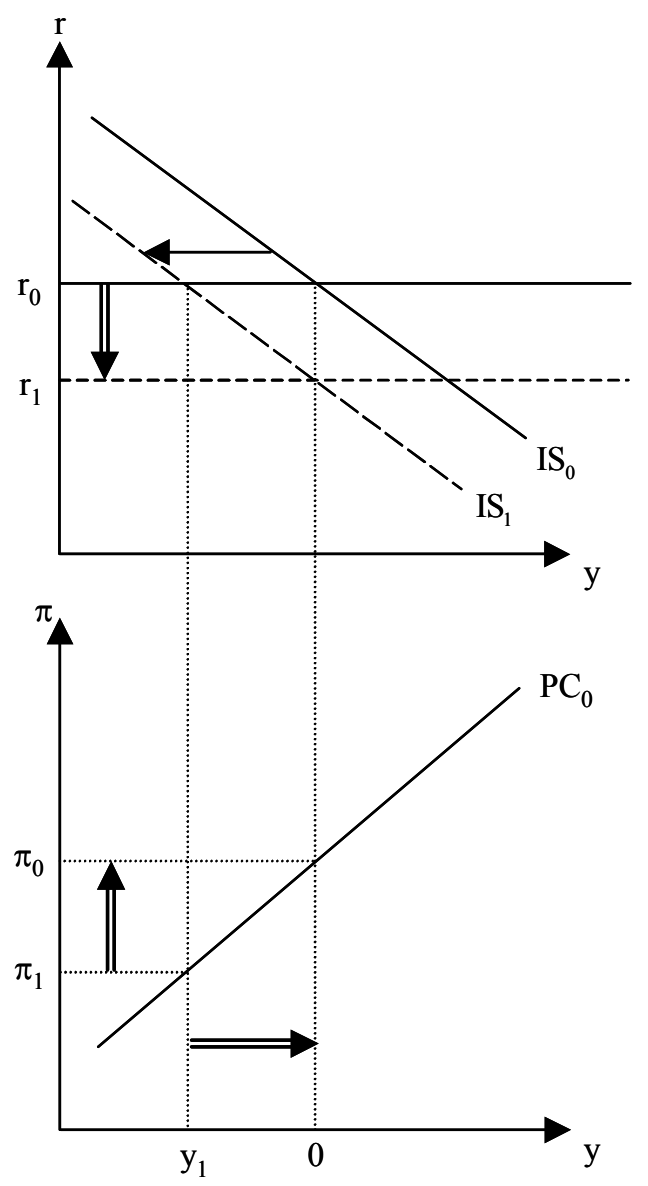

Abb. 1: Nachfrageschocks und diskretionäre Geldpolitik

Bei einem positiven Angebotsschock $\left(\varepsilon_{2}>0\right)$ verschiebt sich die Phillipskurve PC im unteren Teil der Grafik nach oben (vgl. Abb. 2). Im oberen Teil der Grafik kann man erkennen, dass durch einen konstant gehaltenen Realzins $r_{B}=r_{0}$ eine Outputlücke vermieden wird. Allerdings steigt die Inflationsrate von $\pi_{0}$ auf $\pi_{\mathrm{B}}$ (Punkt B). Alternativ kann die Notenbank den Realzins auf $\mathrm{r}_{\mathrm{A}}$ erhöhen, um die Inflationsrate auf dem Zielniveau zu stabilisieren. Abb. 2 zeigt, dass der Preis für dieses Vorgehen eine negative Outputlücke (Punkt A) ist. Zwischen den beiden Extremlösungen $\mathrm{A}$ und $\mathrm{B}$ ist jede Kompromisslösung zwischen der Stabilisierung der Inflationsrate und des Outputs denkbar und möglich. 


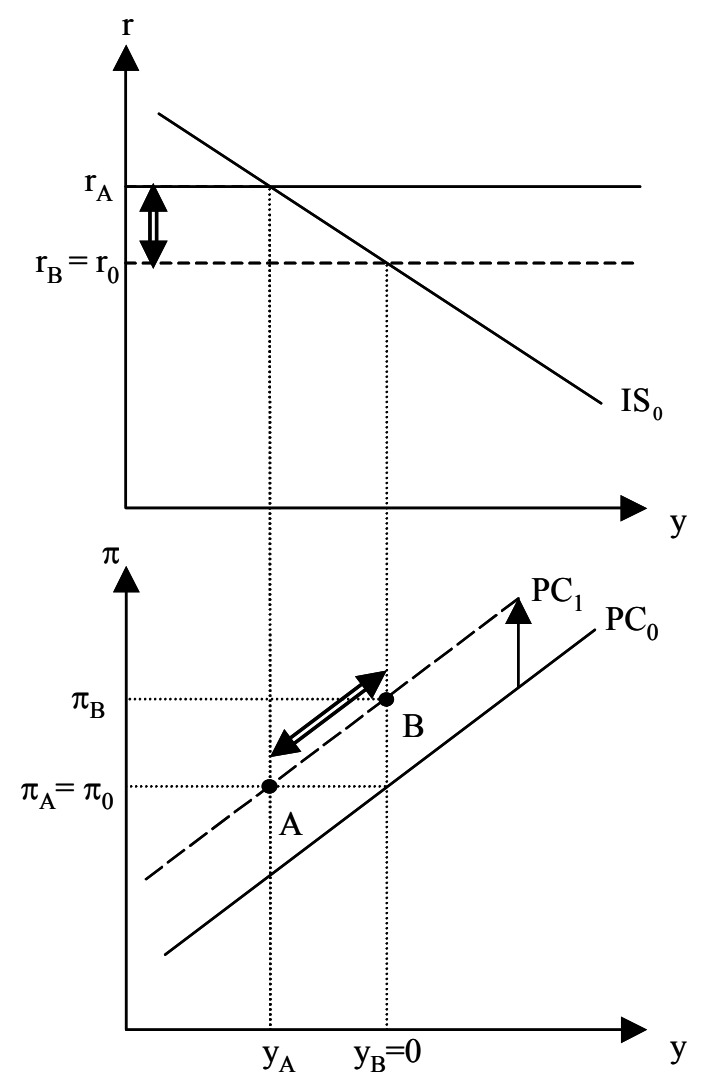

Abb. 2: Angebotsschock und diskretionäre Politik

Diese Basisversion des Modells hilft bereits die Wirkungsweise von Geldpolitik in Grundzügen zu verstehen. Geldpolitik, die auf Angebots- und Nachfrageschocks reagiert, wirkt sich direkt auf den Output aus - wie im r-y-Raum der Abb. zu sehen ist. Ihren Einfluss auf die Inflationsrate entfaltet die Geldpolitik über die Manipulation der Outputlücke, wie sich im $\pi$-y-Raum ablesen lässt. Im Vergleich zum IS/LM-AS/AD Model ist die Basisversion des BMW-Modells

- einfacher, weil keine LM Kurve angepasst werden muss, nachdem ein verändertes Preisniveau die reale Geldnachfrage beeinflusst hat,

- aussagekräftiger, weil es auf die Inflationsrate und nicht auf das Preisniveau abzielt, und

- umfassender durch die um Erwartungen erweiterte Phillipskurve.

Für einführende Lehrveranstaltungen ist das Modell in dieser Form bereits ausreichend und könnte sogar dadurch weiter vereinfacht werden, dass man auf die beiden Schockterme $\varepsilon_{1}$ und $\varepsilon_{2}$ verzichtet. Einen vergleichbaren Ansatz hat bereits Taylor (2001, Kapitel 24) gewählt. 


\subsection{Inflation Targeting}

Eine Regelbindung der Notenbank erleichtert nicht nur den Entscheidungsprozess innerhalb der Notenbank, sondern dient auch der Transparenz gegenüber der Öffentlichkeit und sorgt dafür, dass diese die Entscheidungen ex-post besser beurteilten können. Die inzwischen bekannteste Strategie ist das Inflation Targeting. Diese Strategie kann modelliert werden, indem die Endziele einer Notenbank durch eine eine Verlustfunktion abgebildet werden:

$$
\mathrm{L}=\left(\pi-\pi_{0}\right)^{2}+\lambda \mathrm{y}^{2} \text { mit } \lambda \geq 0
$$

Die Notenbank, die sich an einer solchen Verlustfunktion orientiert, versucht die quadratischen Abweichungen der aktuellen Inflationsrate vom Inflationsziel möglichst gering zu halten. Bei einem $\lambda>0$ achtet sie weiterhin darauf, dass Schwankungen der Outputlücke möglichst gering sind. Deshalb spricht man auch von einem „flexiblen Inflation Targeting“. Falls die Notenbank keine Präferenz für die Stabilisierung des Outputs hat $(\lambda=0)$ spricht man von „strengem Inflation Targeting“ oder von einer „inflation nutter“ (Svensson, 1999). Die jeweiligen Abweichungen von ihrem Zielwert werden quadriert, damit positive und negative Abweichungen gleichermaßen ins Gewicht fallen und sich nicht zu Null saldieren.

Bei gegebenem Transmissionsprozess - vom Realzins über den Output hin zur Inflationsrate kann die optimale Geldpolitik folgendermaßen hergeleitet werden: Man setzt die Phillipskurve (4) in die Verlustfunktion (5) ein und leitet nach y ab. Löst man dann die Ableitung nach y auf, so ergibt sich die optimale Outputlücke:

$$
y=-\frac{d}{\left(d^{2}+\lambda\right)} \varepsilon_{2} .
$$

Diese Vorgehensweise deckt sich mit dem Neukeynesianischen Ansatz, wonach die Notenbank Geldpolitik betreibt, indem sie die Outputlücke steuert. Wenn man nun Gleichung (6) in die Phillipskurve einsetzt, lässt sich eine optimale Abweichung der Inflationsrate von der Zielinflationsrate bestimmen:

$$
\left(\pi-\pi_{0}\right)=\frac{\lambda}{\mathrm{d}^{2}+\lambda} \varepsilon_{2}
$$


Im Einklang mit der grafischen Lösung aus Kapitel 2.2 kann man sehen, dass die Notenbank Nachfrageschocks vollständig durch eine geeignete Realzinsvariation kompensieren kann, nachdem der Schockterm $\varepsilon_{1}$ in den Gleichungen (6) und (7) nicht vorkommt.

Eine Notenbank, die die Strategie des Inflation Targetings verfolgt, optimiert im BMW-Modell annahmegemäß den Realzins unter Berücksichtigung aller vorliegenden Informationen, insbesondere der beiden exogenen Variablen $\varepsilon_{1}$ und $\varepsilon_{2}$. Der optimale Realzinsregel lässt sich bestimmen, indem man Gleichung (5) in (1) einsetzt und nach dem Realzins r auflöst:

$$
\mathrm{r}^{\mathrm{opt}}=\frac{\mathrm{a}}{\mathrm{b}}+\frac{1}{\mathrm{~b}} \varepsilon_{1}+\frac{\mathrm{d}}{\mathrm{b}\left(\mathrm{d}^{2}+\lambda\right)} \varepsilon_{2}
$$

Gleichung (8) wird in Anlehnung an Svensson und Woodford (2003) als Instrumentenregel (,instrument rule“) bezeichnet, da sie das geldpolitische Instrument der Notenbank in Abhängigkeit aller ihr im Zeitpunkt t zur Verfügung stehenden Informationen darstellt.

Es zeigt sich, dass die optimale Reaktion auf Nachfrageschocks $\varepsilon_{1}$ nicht von den Präferenzen $\lambda$ der Notenbank abhängt, sondern das $r$ ausschließlich entsprechend $(1 / b) \varepsilon_{1}$ angepasst wird. Solange die Notenbank einen Nachfrageschock erkennt und quantifizieren kann, verursacht er keine sozialen Kosten im Sinne der Verlustfunktion (5). Im Gegensatz dazu treten bei Angebotsschocks $\varepsilon_{2}$ soziale Kosten auf. Die Reaktion auf Angebotsschocks hängt von $\lambda$ ab. Eine Notenbank, die ausschließlich eine Präferenz für das Erreichen des Inflationsziels hat $(\lambda=0)$, wird den Realzins stark anpassen. Mit zunehmendem $\lambda$ wird die Reaktion auf einen Angebotsschock schwächer ausfallen (vgl. Punkt B in Abb. 2). Im Gleichgewicht $\left(\varepsilon_{1}=\varepsilon_{2}=0\right)$ ist der optimale Realzins gegeben durch $r_{0}=a / b$.

Grafisch ist die Strategie des Inflation Targetings in Abb. 3 dargestellt. Die ,instrument rule“ wird als horizontale Linie im r-y-Raum abgebildet $-\mathrm{r}\left(\varepsilon_{1}, \varepsilon_{2}\right)$. Die Verlustfunktion der Notenbank kann grafisch beschrieben werden durch konzentrische Kreise um den Optimalpunkt im $\pi$-y-Raum. Der Optimalpunkt befindet sich an der Stelle, an der die Outputlücke und die 
Inflationsrate ihren jeweiligen Zielwerten entsprechen. Analytisch kann die Verlustfunktion durch folgende Kreisgleichung dargestellt werden:

$$
1=\frac{\left(\pi-\pi_{0}\right)^{2}}{(\sqrt{\mathrm{L}})^{2}}+\left.\frac{(\mathrm{y}-0)^{2}}{(\sqrt{\mathrm{L}})^{2}}\right|_{\lambda=1}
$$

wobei $\left(0 ; \pi_{0}\right)$ der Mittelpunkt des Kreises ist, und der Radius durch $\sqrt{\mathrm{L}}$ gegeben ist.

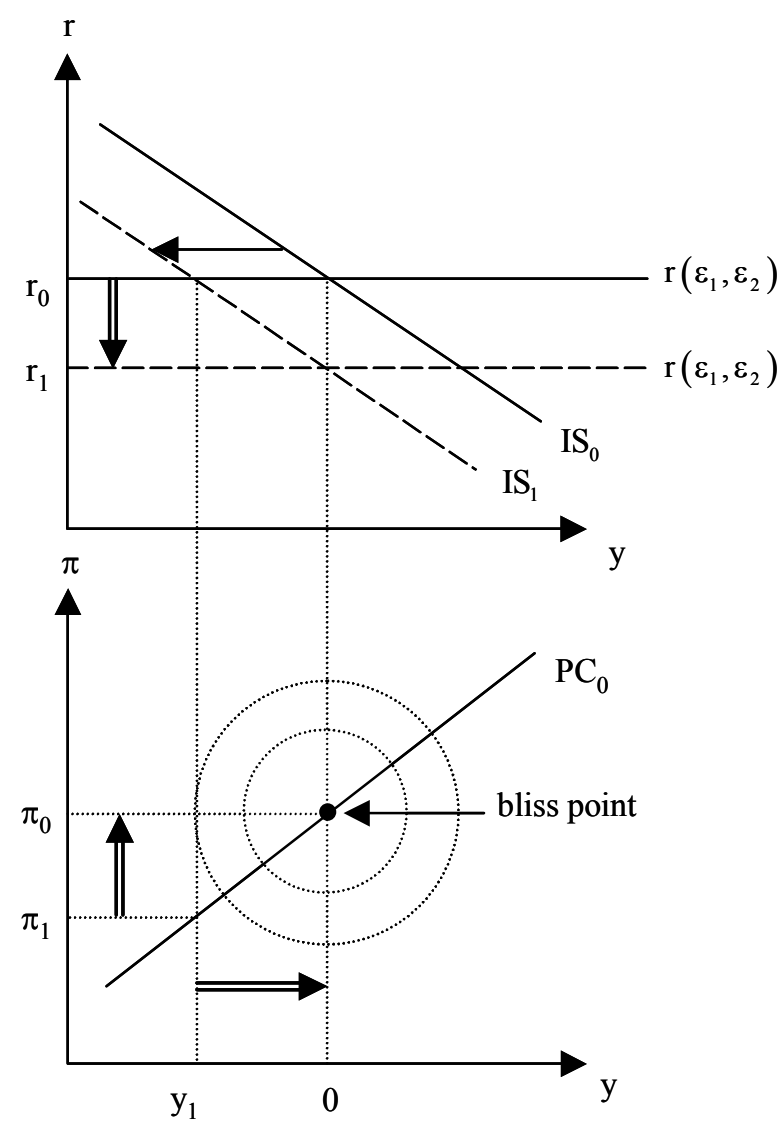

Abb. 3: Nachfrageschock und optimale geldpolitische Reaktion der Notenbank

Im Fall eines Nachfrageschocks ist die Notenbank immer in der Lage, den Optimalpunkt in der Mitte des Kreises zu erreichen (vgl. Abb. 3). Falls ein Angebotsschock auftritt, lässt sich mit der Verlustfunktion die optimale Kombination von $\pi$ und y ermitteln (vgl. Abb. 4). Die nach oben verschobene Phillipskurve dient dabei als Restriktion, unter der der Radius des Kreises minimiert werden muss. Im unteren Teil der Grafik lässt sich die optimale Kombination $\left(\mathrm{y}_{1} ; \pi_{1}\right)$ als 
Tangente der Phillipskurve am Kreis der Verlustfunktion bestimmen. Die adäquate geldpolitische Reaktion der Notenbank wird im r-y-Raum bestimmt.

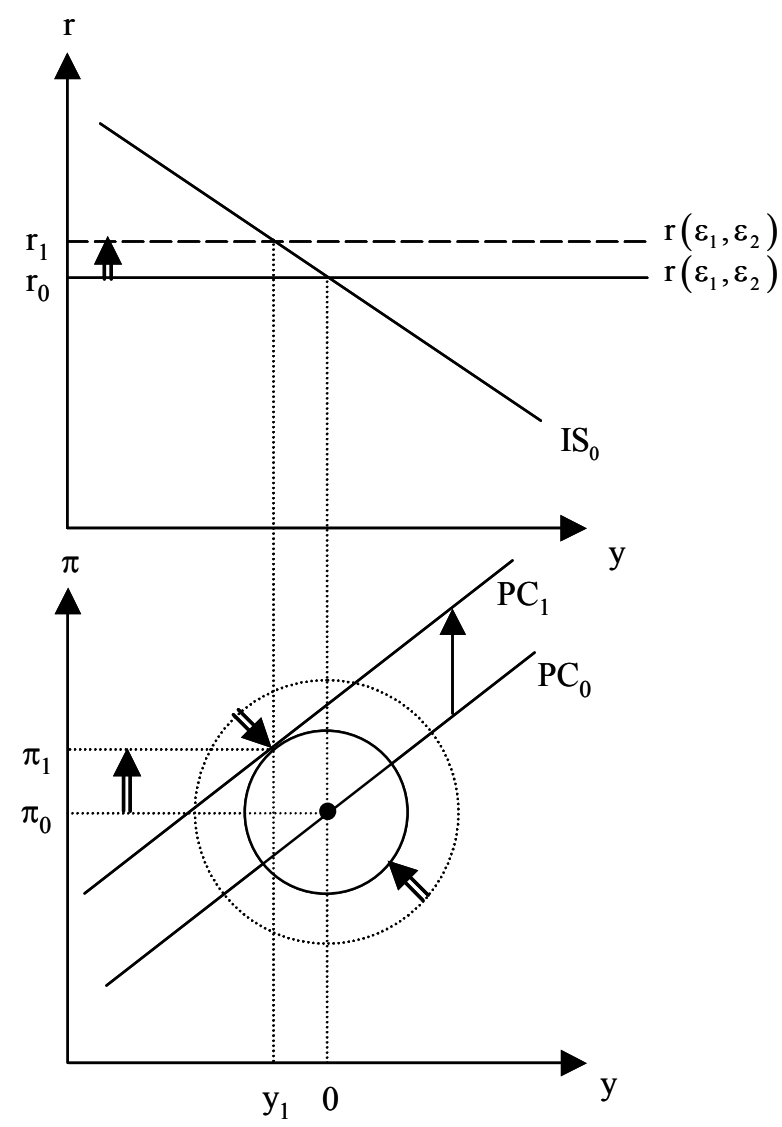

Abb. 4: Angebotsschock und optimale geldpolitische Reaktion der Notenbank

Der geometrische Ort aller optimalen Kompromisslösungen zwischen $\pi$ und y lässt sich in Gleichungsform darstellen, indem man aus Gleichungen (6) und (7) den Schockterm $\varepsilon_{2}$ eliminiert:

$$
\pi=\pi_{0}-\frac{\lambda}{\mathrm{d}} \mathrm{y}
$$

Bei Gleichung (10) handelt es sich gemäss Svensson und Woodford (2003) um die sogenante Reaktionsfunktion (RF) der Notenbank. Man kann diese Regel auch als „targeting rule“ bezeichnen, da sie eine Linearkombination zwischen den Zielgrössen der Geldpolitik darstellt (Inflation und Outputlücke), die bei optimaler Geldpolitik erfüllt sein muss. Gleichung (10) zeigt, dass ein steigendes $\lambda \mathrm{zu}$ einer steileren Reaktionsfunktion führt. In der grafischen Lösung 
beschreibt der Schnittpunkt der Phillipskurve $\mathrm{PC}_{1}$ mit der Reaktionsfunktion der Notenbank den optimalen Output (vgl. Abb. 5).

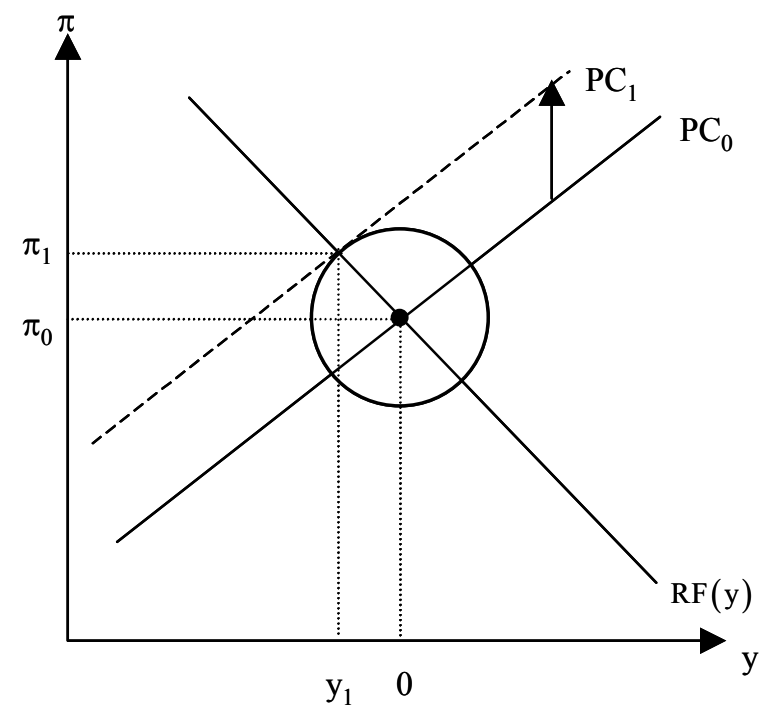

Abb. 5: Zielpunkt der Notenbank und geldpolitische Reaktionsfunktion

\subsection{Die Taylor Regel: Geldpolitik anhand einer einfachen Regel}

Von einer einfachen Regel spricht man, wenn sich die Notenbank an einigen wenigen Größen orientiert, die sie direkt beobachten kann. Die weitläufigst verwandte unter den einfachen Regeln ist die Taylor Regel (Taylor, 1993). Nach dieser Regel hängt der aktuelle Realzinssatz vom kurzfristigen neutralen Realzins $\left(\mathrm{r}_{0}\right)$ und der aktuellen konjunkturellen Situation ab, repräsentiert durch die Abweichung der Inflationsrate von der Zielinflationsrate und von der Outputlücke y:

$$
\mathrm{r}=\mathrm{r}_{0}+\mathrm{e}\left(\pi-\pi_{0}\right)+\text { fy mit e, } \mathrm{f}>0 \text {. }
$$

Im vorliegenden Modell lässt sich die Taylor Regel durch eine Gerade (MP) im r-y-Raum mit positiver Steigung darstellen (vgl. Abb. 6). Während Veränderungen der Outputlücke eine Anpassung des Realzinses durch die Bewegung auf der MP Linie erfordern, ist die Inflationsrate selbst ein Lageparameter.

Im Gegensatz zur Vorgehensweise in den Kapiteln 2.2 und 2.3 ist hier die Konstruktion einer von der Inflationsrate abhängigen Nachfragekurve (AD Kurve) notwendig. Analytisch kann die AD- 
Kurve leicht hergeleitet werden, indem man die Taylor Regel (11) in die IS Kurve (1) einsetzt, $\mathrm{r}_{0}$ durch a/b ersetzt und nach $\pi$ auflöst:

$$
\pi=\pi_{0}+\frac{\varepsilon_{1}}{\mathrm{be}}-\frac{1+\mathrm{bf}}{\mathrm{be}} \mathrm{y} .
$$

Die grafische Konstruktion entspricht der einer Nachfragekurve im AS/AD-Modell. Man beginnt im Gleichgewicht, d.h. mit der MP( $\left.\pi_{0}\right)$ Linie und einer Outputlücke von Null (vgl. $A b b$. 6). Aus dieser Kombination von Output und Inflation ergibt sich der Punkt A im unteren Teil der Abbildung. Anschließend zeichnet man eine zweite MP Linie für eine höhere Inflationsrate $\pi_{1}>\pi_{0}$ ein. Denn nach dem „Taylor-Prinzip“ (Taylor, 1999) soll der Realzins angehoben werden, wenn die Inflationsrate ansteigt (vgl. Gleichung (11)). Das neue Gleichgewicht ist mit einer negativen Outputlücke in Höhe von $\mathrm{y}_{1}$ verbunden. Aus der Kombination von $\mathrm{y}_{1}$ und $\pi_{1}$ ergibt sich der Punkt B im $\pi$-y-Raum. Verbindet man die beiden Punkte A und B miteinander, erhält man die aggregierte Nachfragekurve $\mathrm{AD}_{0}$.

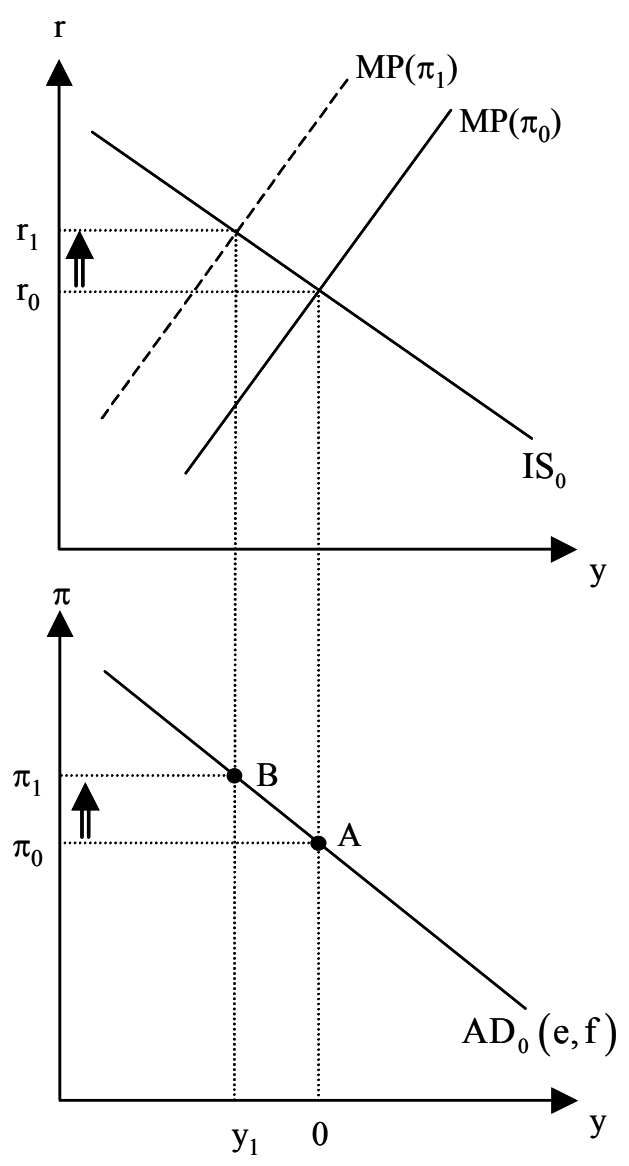


Durch die AD-Kurve wird die Analyse geldpolitischer Maßnahmen etwas komplizierter als im Falle des Inflation Targeting. Wenn - wie in Abb. 7 dargestellt - ein negativer Nachfrageschock auftritt, verschiebt sich die IS Kurve im r-y-Raum nach unten. Auf die daraus resultierende Outputlücke (y') reagiert die Notenbank, indem sie den Realzins senkt. Grafisch entspricht das einer Bewegung entlang der MP $\left(\pi_{0}\right)$-Linie von $\mathrm{r}_{0}$ nach r'. Im unteren Teil der Abb. 7 verschiebt sich die AD Kurve nach unten, denn sie muss durch den Punkt A verlaufen, der sich aus dem Schnittpunkt der zunächst unveränderten Inflationsrate und der negativen Outputlücke ergibt. Das neue Gleichgewicht ergibt sich im Punkt B aus dem Schnittpunkt der verschobenen AD Kurve und der unveränderten Phillipskurve. Im Punkt B ist die Outputlücke mit $\mathrm{y}_{1}$ nicht ganz geschlossen, aber sie ist kleiner als y', und die Inflationsrate liegt mit $\pi_{1}$ durch die Zinssenkung der Notenbank wieder näher an ihrem Zielwert. Das liegt daran, dass die Notenbank den Realzins erneut wegen der gesunkenen Inflationsrate $\left(\pi_{0}\right.$ auf $\left.\pi^{\prime}\right)$ anpassen musste - jetzt von $r^{\prime}$ auf $r_{1}$. Im oberen Teil der Abb. 7 wird dies durch die Verschiebung der MP Kurve zum Ausdruck gebracht, so dass sie die $\mathrm{IS}_{1}$ Kurve im Punkt $\left(\mathrm{r}_{1} ; \mathrm{y}_{1}\right)$ schneidet. Die Vorgehensweise ist die gleiche wie im IS/LM-AS/AD-Modell im Fall eines Nachfrageschocks. Während im vorliegenden Modell die gesunkene Inflationsrate einen expansiven Effekt hat, nachdem dadurch der Realzins sinkt, verursacht im IS/LM-AS/AD-Modell das gesunkene Preisniveau eine steigende Geldmenge was ebenfalls expansiv wirkt. 


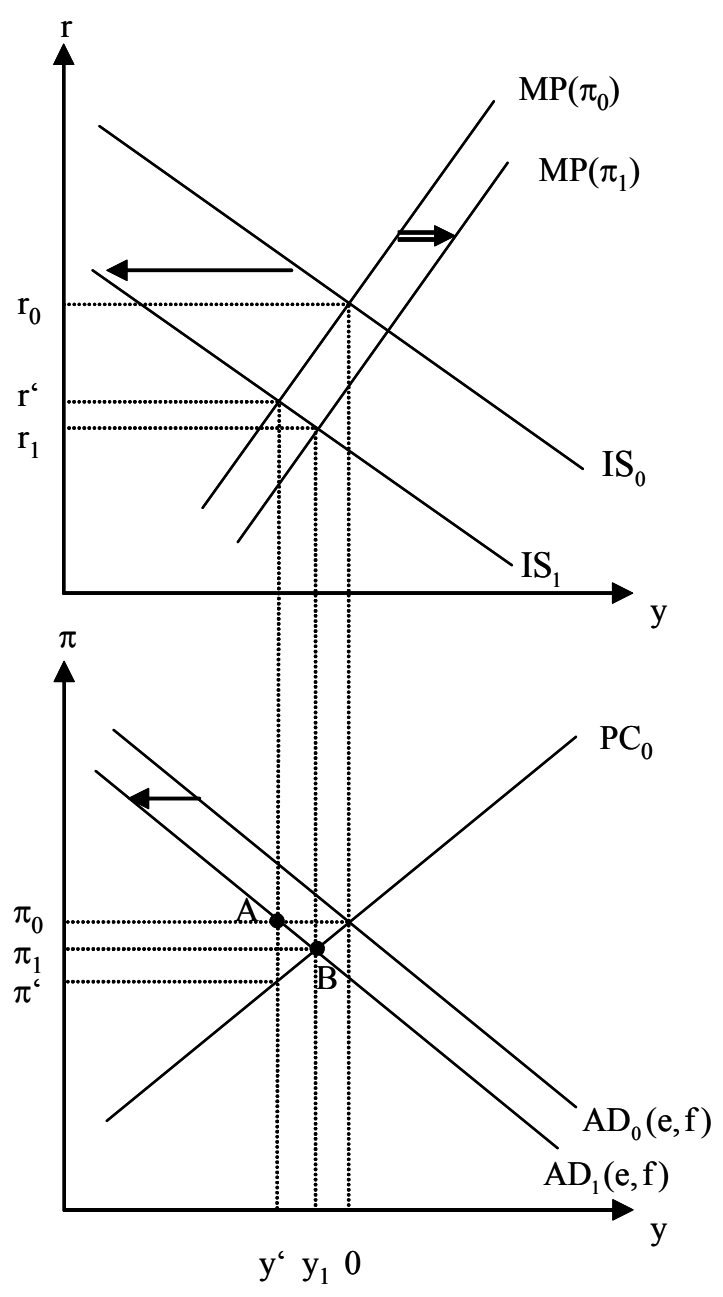

Abb. 7: Nachfrageschocks und Taylor-Regeln

Für die grafische Analyse geldpolitischer Maßnahmen nach einem Angebotsschocks genügt es, wenn man sich ausschließlich den $\pi$-y-Raum anschaut (Abb. 8). Dem Angebotsschock entspricht eine Verschiebung der Phillipskurve nach oben. Dadurch steigt die Inflationsrate. Die TaylorRegel verlangt nun eine Erhöhung des Realzinses, wodurch eine negative Outputlücke entsteht. Die negative Outputlücke wiederum dämpft leicht den Anstieg der Inflationsrate auf $\pi_{1}$. 


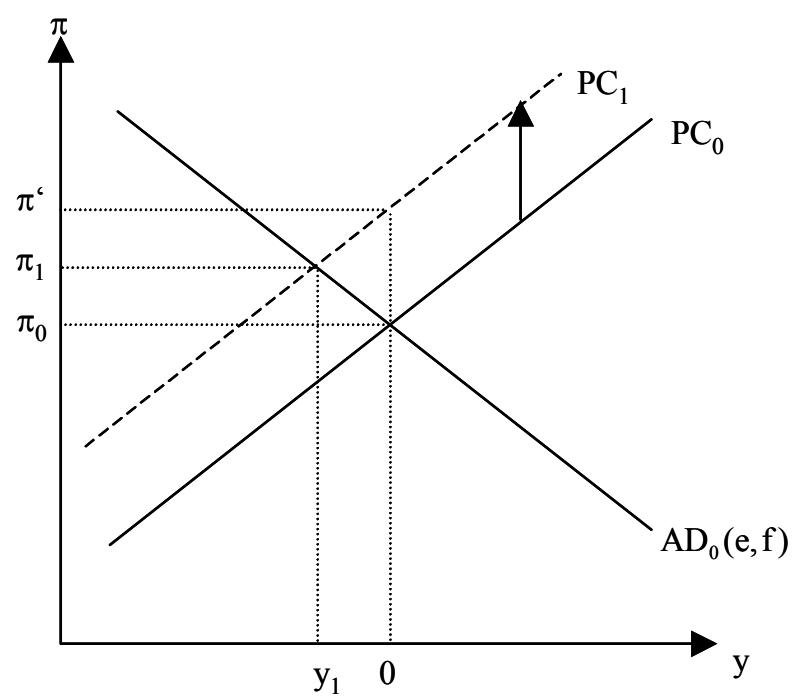

Abb. 8: Einfache Regeln und Angebotsschocks

Abschließend wollen wir einige Bemerkungen zu den Koeffizienten in der Reaktionsfunktion der Notenbank machen: Ein höheres Gewicht auf die Veränderung der Outputlücke - also ein höheres $\mathrm{f}$ - führt zu einer steileren AD Kurve und gleichzeitig zu einer kleineren Outputlücke. Ein größeres e - also eine größere Gewichtung der Inflationsrate - flacht die AD Kurve ab und führt somit zu kleineren Abweichungen der Inflationsrate von ihrem Zielwert. Die Taylor Regel führt zu sinnvollen Ergebnissen, solange e $>0$ ist. Wenn e $<0$ ist hat die AD Kurve eine positive Steigung und Geldpolitik wirkt destabilisierend, da sie Schocks verstärkt (Abb. 9). 


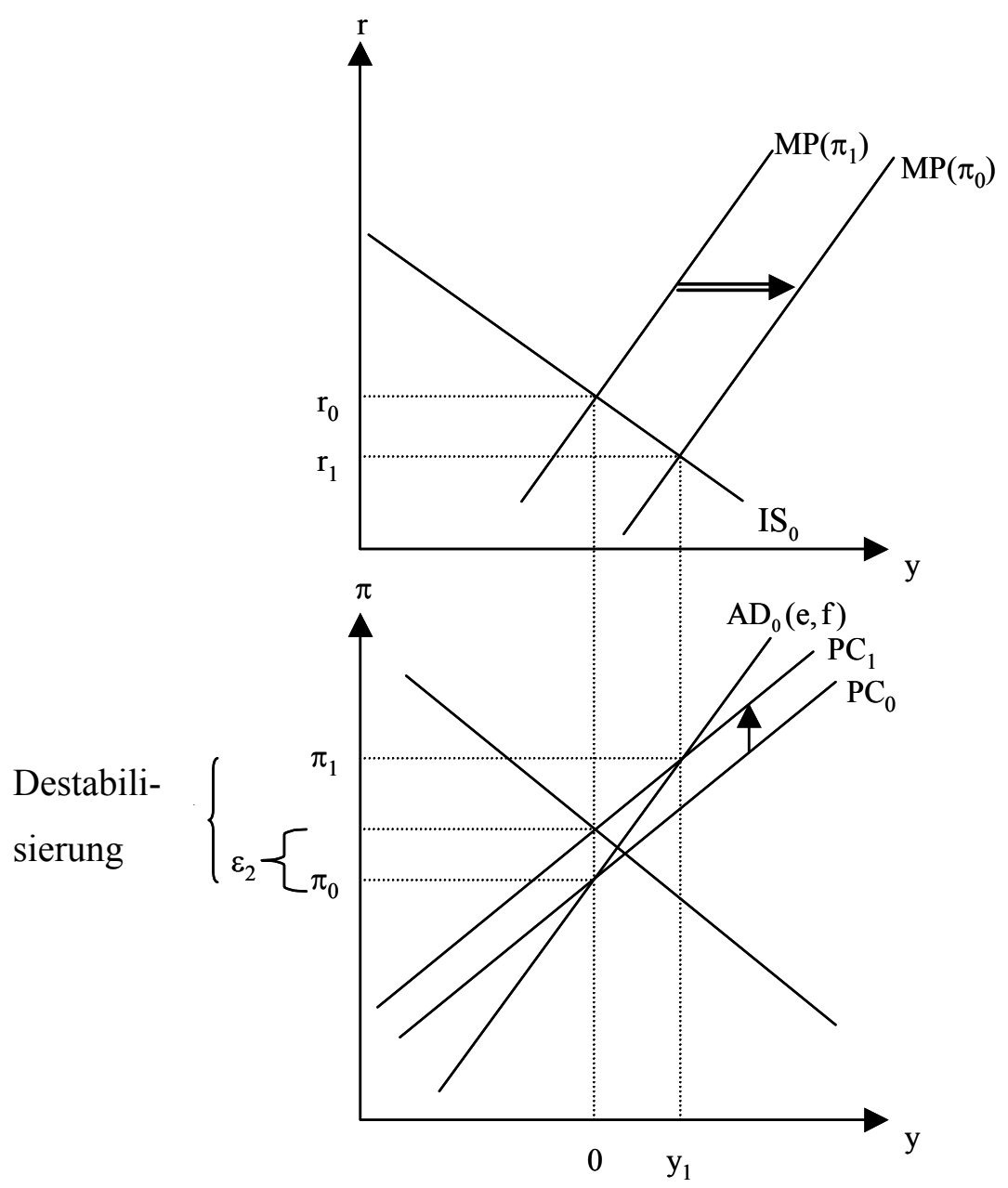

Abb. 9: Destabilisierende Effekte einer "passiven” Taylor Regel

\subsection{Rationale Erwartungen im BMW-Modell}

In diesem Kapitel wollen wir die Erwartungen über die Inflationsrate $\pi^{\mathrm{e}}$, wie sie in der allgemeinen Form der Phillipskurve (3) zu finden sind, endogenisieren. Wir wollen zeigen, dass die Vereinfachung $\pi^{\mathrm{e}}=\pi_{0}$, die wir in Kapitel 2.1 vorgenommen haben, allgemeingültig ist, wenn die Privaten ihre Erwartungen rational bilden. Es wird wiederum unterstellt, dass die Notenbank ihre Geldpolitik an der Verlustfunktion (5) ausrichtet und dass die Struktur der Volkswirtschaft durch die Gleichungen (1) und (3) beschrieben wird. Setzt man die Phillipskurve in die Verlustfunktion ein und minimiert man diese, so erhält man die optimale Outputlücke:

$$
y=-\frac{d}{d^{2}+\lambda}\left(\pi^{e}-\pi_{0}\right)-\frac{d}{d^{2}+\lambda} \varepsilon_{2}
$$


Durch Einsetzen von Gleichung (13) in die Phillipskurve lässt sich die optimale Inflationsrate der Notenbank als eine Funktion der Inflationserwartungen des privaten Sektors darstellen:

$$
\pi\left(\pi^{\mathrm{e}}\right)=\frac{\lambda}{\mathrm{d}^{2}+\lambda} \pi^{\mathrm{e}}+\frac{\mathrm{d}^{2}}{\mathrm{~d}^{2}+\lambda} \pi_{0}+\frac{\lambda}{\mathrm{d}^{2}+\lambda} \varepsilon_{2}
$$

Am Beginn einer jeden Periode bilden die Privaten Erwartungen über die Inflationsrate, die in der Periode herrschen wird. Diese Erwartungen fließen in die von ihnen geschlossenen Verträge auf den Güter- und Arbeitsmärkten ein. Es sei nun unterstellt, dass die Privaten darauf abzielen, die Abweichungen der tatsächlichen Inflationsrate von der erwarteten zu minimieren. Leitet man ihre Verlustfunktion

$$
\mathrm{L}^{\text {priv }}=\left(\pi\left(\pi^{\mathrm{e}}\right)-\pi^{\mathrm{e}}\right)^{2}
$$

nach der Inflationsrate $\pi\left(\pi^{\mathrm{e}}\right)$ ab, ergibt sich folgende optimale Inflationsrate:

$$
\pi^{\mathrm{opt}}\left(\pi^{\mathrm{e}}\right)=\pi^{\mathrm{e}}
$$

Unter der Annahme rationaler Erwartungen nutzen die Privaten nun alle ihnen zur Verfügung stehenden Informationen, insbesondere die optimale Inflationsrate der Notenbank. Setzt man Gleichung (14) in Gleichung (16) ein,

$$
\pi^{\mathrm{e}}=\frac{\lambda}{\mathrm{d}^{2}+\lambda} \pi^{\mathrm{e}}+\frac{\mathrm{d}^{2}}{\mathrm{~d}^{2}+\lambda} \pi_{0}+\frac{\lambda}{\mathrm{d}^{2}+\lambda} \varepsilon_{2}
$$

und löst man diese Gleichung nach $\pi^{\mathrm{e}}$ auf, erhält man

$$
\pi^{\mathrm{e}}=\pi_{0}
$$

unter der Annahme, dass der Angebotsschock $\varepsilon_{2}$ zu Beginn der betrachteten Periode noch nicht aufgetreten ist, und somit auch nicht Bestandteil der Informationsmenge der Privaten ist. Dieser Modellansatz kann sehr einfach erweitert werden und erlaubt erlaubt ebenfalls die Modellierung einer Zentralbank mit einem Glaubwürdigkeitsproblem (Barro und Gordon, 1983). Zu diesem Zweck müssen wir die Verlustfunktion wie folgt modifizieren:

$$
\mathrm{L}=\left(\pi-\pi_{0}\right)^{2}+\lambda(\mathrm{y}-\mathrm{k})^{2} \text { with } \mathrm{k}>0
$$


Durch die Einführung des k-Faktors lässt sich eine überambitionierte Notenbank abbilden, die eine Outputlücke größer als Null erreichen will. Diese Zielsetzung lässt sich zum Beispiel mit Hilfe vom monopolitischen Verwerfungen auf Güter und Faktormärkten begründen, die den Output unterhalb des Vollbeschäftigungseinkommens absinken lassen. Basierend auf dieser Verlustfunktion können nun ebenfalls die weitläufig bekannten Ergebnisse des Barro und Gordon Modells (1983) abgeleitet werden (siehe Bofinger, Mayer, Wollmershäuser (2002a)).

\section{Schlussfolgerungen}

Zusammenfassend bietet unser Modell also entscheidende Vorteile gegenüber dem IS/LMAS/AD-Ansatz. In seiner Grundversion ist es sowohl einfacher aber auch überzeugender zur Darstellung grundlegender Sachverhalte als das IS/LM-AS/AD-Modell. In seiner erweiterten Version kann es angewandt werden um moderne Konzepte wie Inflation Targeting und geldpolitische Regeln zu veranschaulischen. Verglichen mit anderen Ansätzen (Romer, 2000; Taylor, 2001; Walsh, 2002) modellieren wir explizit die Reaktion der Notenbank auf Nachfrageschocks. Desweiteren stellen wir Konzepte wie Glaubwürdigkeit die eine zentrale Rolle im Rahmen des Inflation-Targetings spielen dar. Darüberhinaus ermöglicht das BMWModell einen guten Vergleich zwischen optimaler und einfacher Geldpolitik der im Rahmen eines statischen Ansatzes eine Neuerung darstellt. 


\section{References}

Barro, R. J., and D. B. Gordon. 1983. A positive theory on monetary policy in a natural rate model. Journal of Political Economy 91 (August): 589-610.

Blinder, A. S. 1998. Central Banking in Theory and Practice. Cambridge, Mass.: MIT Press.

Bofinger, P., E. Mayer, and T. Wollmershäuser 2002a. The BMW model: a new framework for teaching monetary macroeconomics in closed and open economies. Würzburg Economic Papers No. 34.

Bofinger, P., E. Mayer, and T. Wollmershäuser 2002b. The BMW model: simple macroeconomics for closed and open economies - a requiem for the IS/LM-AS/AD and the Mundell-Fleming model. Würzburg Economic Papers No. 35.

Bofinger, P., and E. Mayer 2003a. Monetary and Fiscal Policy Interaction in the Euro Area with different Assumptions on the Phillips Curve. Würzburg Economic Papers No. 40.

Bofinger, P., E. Mayer, and T. Wollmershäuser 2003b. The BMW model as a static approximation of a forward-looking New Keynesian macroeconomic model. Würzburg Economic Papers No. 42.

Calvo, G. A. 1983. Staggerred Prices in a Utility-Maximizing Framework, in. Journal of Monetary Economics 12: 383-398.

Clarida, R., Galí, J. and M. Gertler 1999. The Science of Monetary Policy: A New Keynesian Perspective. Journal of Economic Literature (December):1661-1707.

Colander, D. 1995. The Stories we Tell: A Reconsideration of AS/AD Analysis. Journal of Economic Perspectives 9 (Summer): 169-188. 
Romer, D. 2000. Keynesian Macroeconomics without the LM curve. Journal of Economic Perspectives 14 (Summer): 149-169.

Svensson, L. E. O. 1999. Inflation targeting as a monetary policy rule. Journal of Monetary Economics 43 (June): 607-654.

Svensson, L. E.O. and M. Woodford 2003: Implementing Optimal Policy through InflationForecast Targeting. NBER Working Paper no. 9747.

Taylor, J. B. 2001. Economics, $3^{\text {rd }}$ edition: Houghton Mifflin Company, New York, NY.

Taylor, J. B. 1999. A Historical Analysis of Monetary Policy Rules. John B. Taylor, ed. Chicago: Chicago U. Press.

Taylor, J. B. 1993. Discretion versus Policy Rules in Practice. Carnegie-Rochester Conference Series on Public Policy 39 (December): 195-214.

Walsh, C. E. 2002. Teaching Inflation Targeting: An Analysis for Intermediate Macro. The Journal of Economic Education 33 (Fall): 333-346. 